\title{
Dust formation of Be stars with large infrared excess
}

\author{
Chien-De Lee ${ }^{1}$ and Wen-Ping Chen ${ }^{1,2}$ \\ ${ }^{1}$ Graduate Institute of Astronomy, National Central University, \\ Jhongli 32001, Taiwan \\ email: m959009@astro.ncu.edu.tw \\ ${ }^{2}$ Department of Physics, National Central University, \\ Jhongli 32001, Taiwan \\ email: wchen@astro.nuc.edu.tw
}

\begin{abstract}
Classical Be stars, in addition to their emission-line spectra, are associated with infrared excess which is attributable to free-free emission from ionized gas. However, a few with exceptionally large near-infrared excess, namely with $\mathrm{J}-\mathrm{H}$, and $\mathrm{H}-\mathrm{K}_{\mathrm{s}}$ both greater than $0.6 \mathrm{mag}$ - and excess emission extending to mid- and far-infrared wavelengths - must be accounted for by thermal emission from circumstellar dust. Evolved Be stars on the verge of turning off the main sequence may condense dust in their expanding cooling envelopes. The dust particles should be very small in size, hence reprocess starlight efficiently. This is in contrast to Herbig Ae/Be stars for which the copious infrared excess arises from relatively large grains as part of the surplus star-forming materials.
\end{abstract}

Keywords. stars: early-type, stars: evolution, stars: emission-line, Be, stars: pre-main-sequence, infrared: stars

\section{Introduction}

When the first emission-line star was detected by Padre Angelo Secchi in 1866 (Porter \& Rivinius 2003), the term "Be stars" referred to a general class of early-type stars exhibiting emission lines in their spectra. After about a hundred years, early-type premain sequence stars, also with emission-line spectra, were identified by Herbig (1960) and later called Herbig Ae/Be (HABe) stars. To distinguish from HABe stars and giant emission-line stars, the early-type main-sequence stars with emission lines are called classical Be $(\mathrm{CBe})$ stars.

$\mathrm{CBe}$ stars are fast rotators, with a near-critical equatorial speed. They also show infrared (IR) emission in excess of photospheric radiation, often attributed to free-free emission from ionized gas in an extended circumstellar envelope. This is thought to differ from the large IR excess seen in HABe stars, which arises from reprocessing of starlight by circumstellar dust left over from star formation. A few CBe stars show abnormally large IR excess, yet appear not to belong to a young stellar population. We present their IR properties and discuss the possible dust formation mechanism.

\section{Infrared excess}

The left panel of Fig. 1 shows where typical HABe, B[e], and CBe stars occupy in a 2MASS J-H versus H-Ks diagram. Compared with HABe or $\mathrm{B}[\mathrm{e}]$ stars, the majority of CBe stars appear only slightly redder, with $\mathrm{J}-\mathrm{H} \lesssim 0.1$ and $\mathrm{H}-\mathrm{K}_{\mathrm{s}} \lesssim 0.2$, than the earlytype main sequence locus. A few CBe stars, however, exhibit near-IR excess as large as that of HABe stars, having $(\mathrm{J}-\mathrm{H})$ and $\left(\mathrm{H}-\mathrm{K}_{\mathrm{s}}\right) \sim 0.6-0.7 \mathrm{mag}$. 

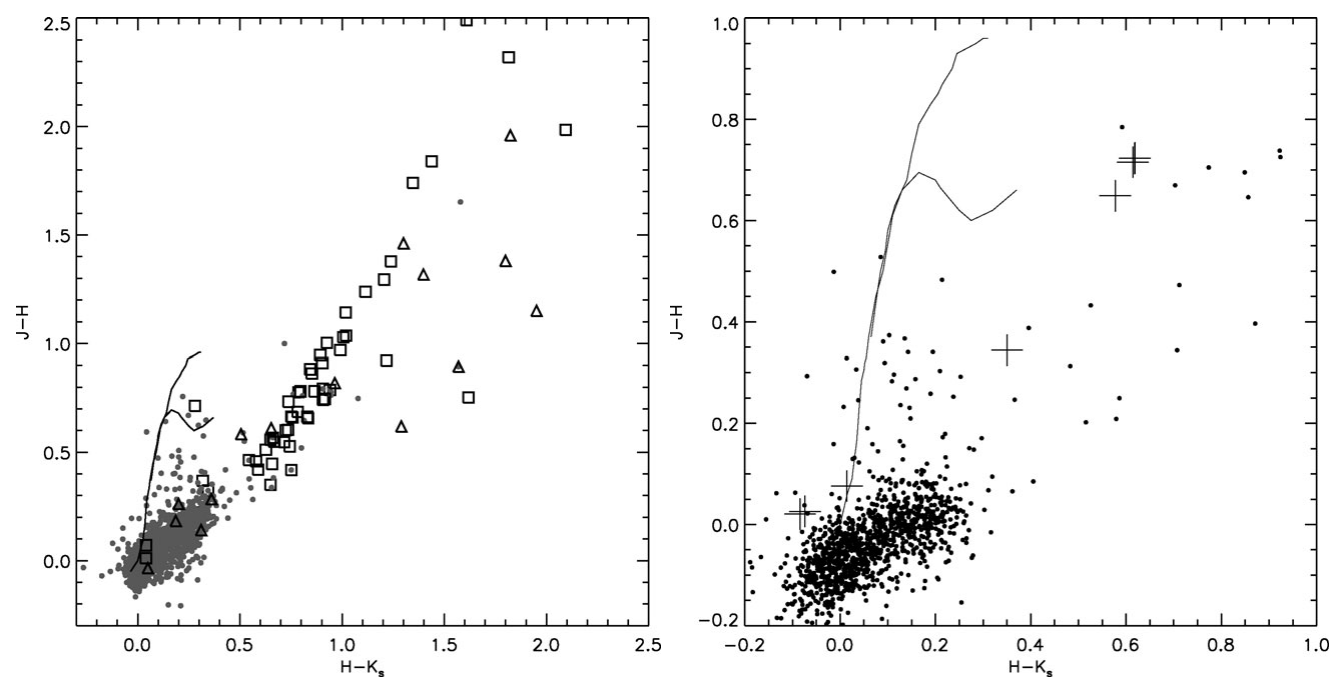

Figure 1. (Left): In the 2MASS J-H versus $\mathrm{H}-\mathrm{K}_{\mathrm{s}}$ diagram, most $\mathrm{CBe}$ stars (grey dots) are distinctly separated from Herbig Ae/Be (squares) and B[e] stars (trangles). The sample of $\mathrm{CBe}$ stars is taken from Zhang, Chen \& Yang (2005), and data of both Herbig Ae/Be and B[e] stars are taken from de Winter et al. (2001). (Right): The same color-color diagram but only CBe stars are shown. The crosses indicate how free-free emission with different levels of electron number density and emitting size affects the observed near-IR colors of stars. From bottom left, each cross represents an increase of an order, from $10^{\circ}$ to $10^{6}$ times in emissivity (see text) surrounging a B0 star with a gas temperature of 20,000 K (Gehrz \& Hackwell 1974).

The intensity of free-free emission from an ionized gas is proportional to the square of the electron density $\left(n_{e}^{2}\right)$ and the volume of the emitting gas $\left(R_{g}^{3}\right)$. For CBe stars, typical values $R_{g}=10^{12}-10^{13} \mathrm{~cm}$ and $n_{e}=10^{11}-10^{12} \mathrm{~cm}^{-3}$ (Gehrz \& Hackwell 1974). The right panel of Fig. 1 illustrates the extent to which free-free emission from a hot plasma alters the position of a star, here assuming a B0 photosphere, in the diagram. Setting $R_{0}=10^{12} \mathrm{~cm}$ and $n_{0}=10^{11} \mathrm{~cm}^{-3}$, each cross represents an order increase, from bottom left of $10^{0} n_{0}^{2} R_{0}^{3}$ moving upwards and to the right until $10^{6} n_{0}^{2} R_{0}^{3}$. The last two crosses, for $10^{5}$ and $10^{6}$ times of $n_{0}^{2} R_{0}^{3}$ - unphysically large for a CBe star - are already close together; in fact any increment in density or volume sees nothing but an asymptotic approach to $\mathrm{J}-\mathrm{H} \approx 0.7$, and $\mathrm{H}-\mathrm{K}_{\mathrm{s}} \approx 0.6$.

The fact that free-free emission from a stellar envelope has this behavior is understood as follows. When the contribution of free-free emission is weak, the observed spectral energy distribution (SED), i.e., the addition of the radiation from the photosphere and from the envelope, is only slightly modified from the photospheric (blackbody) radiation. This is seen in most CBe stars. As the free-free emission, which has a fixed frequency dependence, becomes progressively prominent, it dominates the spectral running between $\mathrm{J}$ and $\mathrm{H}$, and between $\mathrm{H}$ and $\mathrm{K}$, thus the fixed $\mathrm{J}-\mathrm{H}$ and $\mathrm{H}-\mathrm{K}_{\mathrm{s}}$ colors. Inclusion of freefree absorption only exacerbates the case. A cool companion may also contribute to near-IR excess. But in no cases would the excess emission extend to mid- or far-IR, or beyond. The only plausible explanation for a large IR excess remain thermal emission from circumstellar dust.

Here we discuss the 6 stars in Fig. 1 which show prominent near-IR excess, namely HD 98922, HD 50138, HD 85567, CD-49 3441, HD 259431 and HD 181615/6. Fig. 2 shows their SEDs, each along with a blackbody fit with a photospheric temperature appropriate for the star, plus a single (maximum) temperature, $T_{d}^{\max }$ fit to the circumstellar dust. The 
inner radius at which $T_{d}^{\max }$ occurs is also labeled in each panel. The excess of HD 98922, HD 50138, HD 85567, and CD-49 3441 extends to mid-IR but they not associated with any obvious star-forming region or nebulocity, as diagnosed by DSS images, each of 1 degree field around each target. Therefore they should not be HABe stars. Whether a pre-main sequence star can be seen in isolation of any nebulocity or other young stars is an open issue. An HABe star, unlike a T Tauri star, lacks the youth signature such the lithium absorption line in the spectrum, so difficult to distinguish from an early-type main-sequence star. The short pre-main sequence lifetime also makes it difficult even for a run-away HABe star to transverse afar from its parental cloud.

HD 259431 could be an HAeBe star, not only because the IR excess has an increasing trend toward far-IR but it is seen against a dark cloud. The IR excess however is smaller than that of a typical HABe star, so it is still possible to be a main-sequence Be star.

HD $181615 / 6$ is a binary which the SED (see Fig. 2) consistent with what Samus et al. (2004) suggested, i.e., with an O9 V star plus a supergiant B8 I with the companion suffering a significant extinction $\left(\mathrm{A}_{\mathrm{V}} \sim 15 \mathrm{mag}\right)$. The supergiant component is believed to be embedded in an envelope (Dudley \& Jeffery 1990), suffering a significant extinction $\left(A_{V} \sim 15 \mathrm{mag}\right)$. Our SED analysis should be taken with caution if the observations at different wavelengths have been collected at separate epochs, so a particular component in the binary might dominate a certain wavelength range in a particular orbital phase. It is nevertheless obvious that while a companion may contribute, at least partly, to the near-IR excess, it cannot account for the increasing excess extending to mid- and far-IR wavelengths. There must ample of dust grains in their atmosphere(s).

\section{Dust formation of evolved Be stars}

The large IR excess of the 6 Be stars must come mainly from circumstellar dust emission. One of them may be HABe stars, for which the dust is part of the surplus material from star formation. These dust grains have grown in dense molecular clouds to micron to sub-micron sizes. At least some of these stars are CBe stars. Schild \& Romanishin (1976) suggested that the fast rotation is the result of conservation of angular momentum, as a CBe star uses up its hydrogen in the contracting core. Singh \& Chaubey (1987) studied some $30 \mathrm{CBe}$ stars and concluded that they are indeed near the turn-off points of the main sequence, in support of the core contraction scheme. As such, a CBe star puffs off mass in a nonspherical fashion, likely in a toroid or disk configuration, because of the fast rotation. The expanding material then cools off and condenses to form dust grains, which reprocess starlight to produce the observed excessive IR emission. The freshly condensed dust grains - in contrast to those in HABe stars — could be tiny, e.g., nano-particles, so are efficient emitters. In particular, the disk geometry allows a CBe star to suffer relatively little optical extinction, as is the case for many CBe stars. The star-disk boundary layer produces the characteristic emission lines, just like in an HABe star.

To delineate the evolutionary status, CBe stars in star clusters are utilized. Slettebak (1985) found CBe stars in two open clusters, NGC 3766 and NGC 4755, to be at any stage on the main sequence. In a sample of some 100 CBe stars in about 50 star clusters, Fabregat \& Torrejon (2000) concluded that star clusters younger than $10 \mathrm{Myr}$ tend to lack of CBe stars, with the maximum incidence occurring at ages of 13-25 Myr. This implies that CBe stars already evolve to a late phase on the main sequence. The double clusters $h$ and $\chi$ with an age of 13.5-14 Myr (Currie et al. 2010), is at the stage for an early-B type star to turn off, and most of the CBe stars in the double clusters are, indeed, very close to the end of main sequence. Furthermore, Currie et al. (2008) presented a $\mathrm{JHK}_{\mathrm{s}}$ two-color diagram of $h$ and $\chi$ Per in which a few CBe stars show very large near-IR 

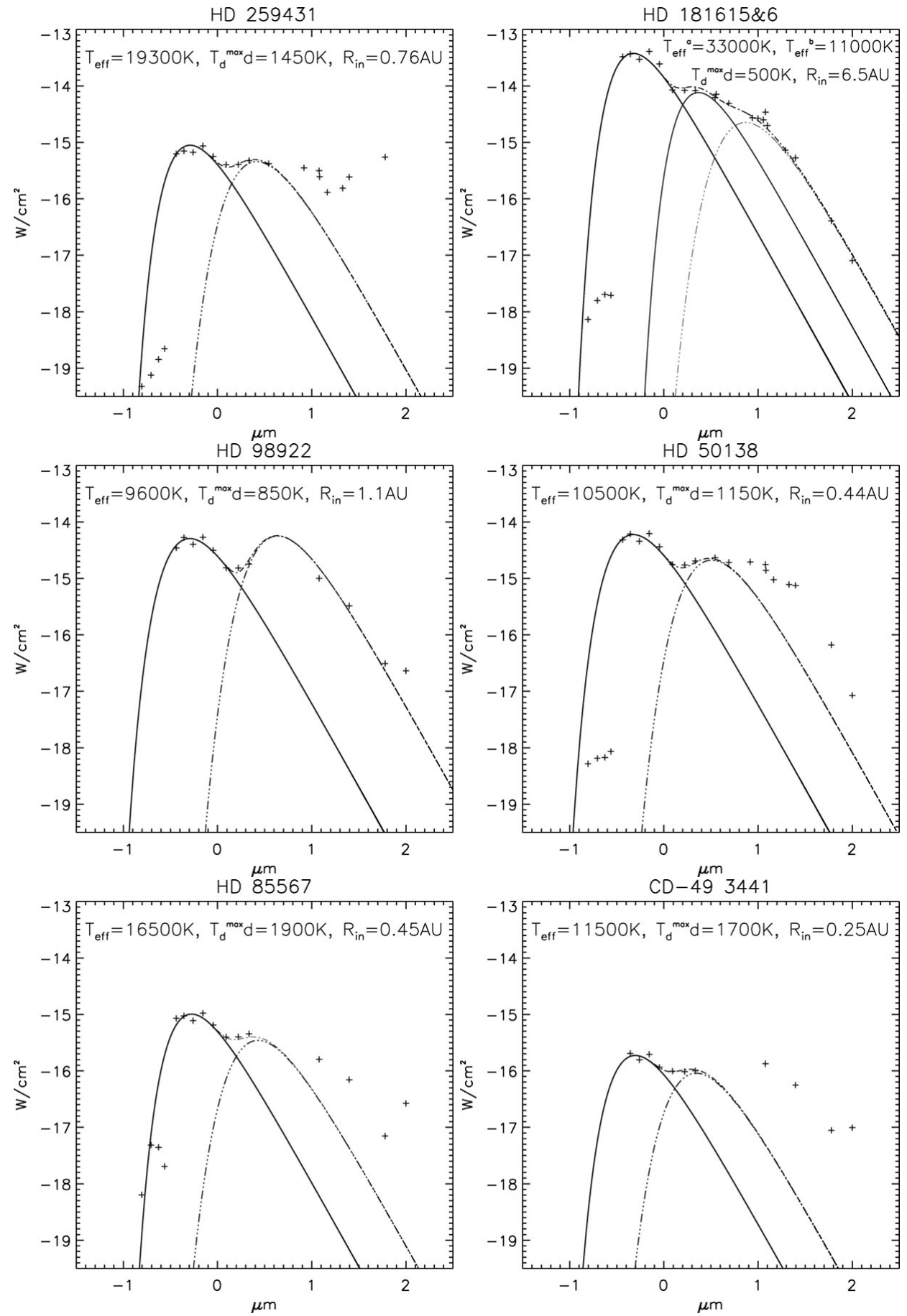

Figure 2. The sample of Be stars show excess emission not only in near-IR, but extends to mid- and far-IR wavelengths.

excess, implying that these CBe stars are just in the beginning of the dust formation. Recently, Mathew et al. (2008) claimed to have identified a CBe star in IC 1590 which is only $\sim 4$ Myr old, marking the nature of CBe stars even more puzzling ever. 


\section{Conclusion}

Most CBe stars show moderate near-IR excess which can be explained by hot circumstellar plasma. Those with IR excess larger than 0.6 mag in both $\mathrm{J}-\mathrm{H}$ and $\mathrm{H}-\mathrm{K}_{\mathrm{s}}$, however, cannot be accounted for by free-free emission, but require thermal emission from circumstellar dust. These dust grains are produced by condensation in the expanding stellar atmosphere, and may have drastically different physical properties from those found around HABe stars.

\section{References}

Currie, T., Hernandez, J., Irwin, J., Kenyon, S. J. et al. 2010, ApJS, 186, 191

Currie, T., Kenyon, S. J., Balog, Z., Rieke, G. et al. 2008, ApJ, 672, 558

Dudley, R. E. \& Jeffery, C. S. 1990, MNRAS, 247, 400

Fabregat, J. \& Torrejón, J. M. 2000, A\& $A$, 357, 451

Gehrz, R. D., Hackwell, J. A. \& Jones, T. W. 1974, ApJ, 191, 675

Herbig, G. H. 1960, ApJS, 4, 337

Mathew, B., Subramaniam, A. \& Bhatt, B. C. 2008, MNRAS, 388, 1879

Porter, J. M. \& Rivinius, T. 2003, PASP, 115, 1153

Samus, N. N, Durlevich, O. V. et al. 2004, Combined General Catalogue of Variable Stars, VizieR On-line Data Catalog: II $/ 250$

Schild, R. \& Romanishin, W. 1976, ApJ, 204, 493

Singh, M. \& Chaubey, U. S. 1987, Ap\&SSS, 129, 251

Slettebak, A. 1985, ApJS, 59, 769

de Winter, D., van den Ancker, M. E., Maira, A., Thé, P. S. et al. 2001, A\&̊A, 380, 609

Zhang, P., Chen, P. S. \& Yang, H. T. 2005, New Astron., 10, 325

\section{Discussion}

Rivinius: Comment: In the $\mathrm{H} \alpha$ of your objects, three have classical P Cygni profiles. This is rather the signature of an expanding wind than that of a rotating disk. Only in the latter case it could be a CBe star.

LEE: Be stars may have stellar winds that produce P Cygni profiles, and at the same time a non-spherical envelope.

WisNiEWSKI: Comment: Just because a star is "isolated" and not associated with a star-forming region does not mean it cannot be a pre-main sequence star. HD 163296, for example is a proto-type of the class of objects known as "isolated HABe stars".

LEE: The existence of many "isolated" HABe stars is not conclusive. In the case of HD 163296, it is actually not isolated, but instead associated with dark clouds, as seen in Fig. 3. It is part of a prominent H II region and a cloud complex.

Miroshnichenko: Comment: The six objects with the large IR excesses presented here do not belong to the class of Be stars. Three of the objects with the $\mathrm{B}[\mathrm{e}]$ phenomenon are members of the FS CMa group, two other are pre-main-sequence HABe stars, and one binary system with a peculiar supergiant. Miroshnichenko et al. (2010, these proceedings) show that circumstellar dust in the FS CMa objects is not fresh (recently formed). The envelopes of Be stars do not contain dust, so these objects need to be excluded from lists of Be stars.

LEE: The term of "classical Be stars" is confusing, to say the least. Any Be stars with circumstellar dust are interesting. Here we present evidence of such dust in at least some 


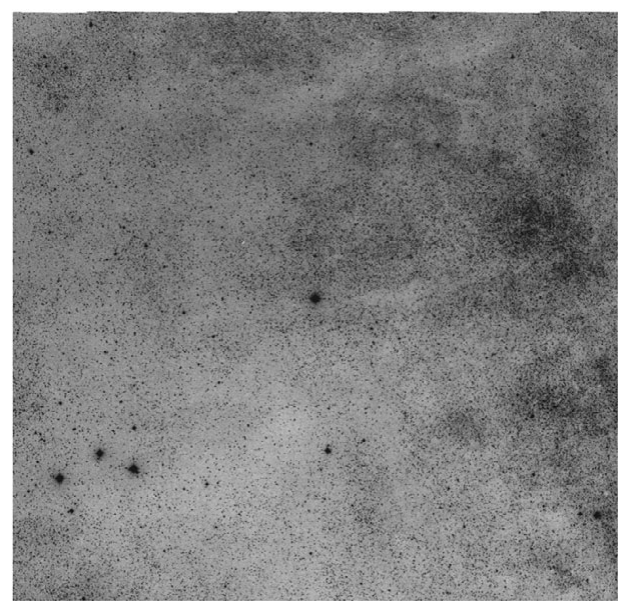

Figure 3. The DSS blue image with HD 163296 at the center. The field is 1 deg square with east to the left and north to the top. Some dark clouds are seen, which is part of prominent emission nebula and dark cloud filaments to the west.

of the CBe stars. The formation, evolution, and properties of these dust grains deserve further investigation. 